\title{
Nefoussa (Djebel) / Adrar n Infusen (« la montagne des Infusen ») : Langue
}

\section{Kamal Naït-Zerrad}

\section{(2) OpenEdition}

\section{Journals}

\section{Édition électronique}

URL : https://journals.openedition.org/encyclopedieberbere/2698

DOI : $10.4000 /$ encyclopedieberbere.2698

ISSN : 2262-7197

\section{Éditeur}

Peeters Publishers

\section{Édition imprimée}

Date de publication : 4 octobre 2012

Pagination : 5363-5369

ISBN : 978-90-429-2640-0

ISSN : 1015-7344

\section{Référence électronique}

Kamal Naït-Zerrad, « Nefoussa (Djebel) / Adrar n Infusen ( "la montagne des Infusen ») : Langue », Encyclopédie berbère [En ligne], 33 | 2012, document N28, mis en ligne le 23 novembre 2020, consulté le 17 février 2022. URL : http://journals.openedition.org/encyclopedieberbere/2698 ; DOI : https:// doi.org/10.4000/encyclopedieberbere.2698

Ce document a été généré automatiquement le 17 février 2022.

(c) Tous droits réservés 


\title{
Nefoussa (Djebel) / Adrar $n$ Infusen (« la montagne des Infusen ») : Langue
}

\author{
Kamal Naït-Zerrad
}

1 On peut distinguer au moins deux groupes de parlers berbères dans ce massif libyen : à partir de l'ouest, celui de Nalut près de la frontière tunisienne, Kabaw, Jadu, Jemmari, etc. d'une part et, à l'autre extrémité, celui de la région de Yefren, d'autre part. Les parlers occidentaux sont ceux étudiés par Beguinot (1942). Dans Motylinski (1898), c'est également dans ces parlers que serait rédigé le «Djebel Nefousa ", même si son auteur est de Yefren. Beguinot et Motylinski précisent que le parler de Yefren est sensiblement différent des parlers occidentaux.

2 On présentera d'abord ces derniers sur la base de l'ouvrage de Beguinot qui décrit en particulier ceux de la région centrale de Fassato (Jadu et Jemmari). De manière générale, ils partagent plusieurs caractéristiques des parlers de Ghadamès (Prasse 1998). Tous les exemples concernant ces parlers proviennent de l'ouvrage de Beguinot.

\section{Phonétique - phonologie - accent}

- Absence de spirantisation des dentales et de labio-vélarisation.

- Au moins une affriquée est attestée comme phonème : $\check{c}[t]$

5 - tendance à l'assourdissement de la dentale pharynagalisée d : ațu «vent », ițan « chiens », ațen « être malade »

6 - Beguinot note assez précisément le timbre des voyelles mais il semble que d'un point de vue phonologique, on retrouve le système des 3 voyelles phonologiques /a/, /i/, /u/ et le schwa [ə] (noté ici «e ») dont le statut n'est pas très clair.

7 Il y aurait un accent - très bien détaillé par Beguinot (1942: 11-18) - et qui serait pertinent dans certains cas (voir également Louali \& Philippson 2005 et Louali 2004). Par exemple, le déplacement de l'accent permettrait de différencier entre : 
rummû « (le) frère » et rûmmu « mon frère »

\section{Nominaux} noms se retrouve dans les parlers nefoussis occidentaux mais elle ne se limite pas, comme ailleurs, à ceux de la forme $(\mathrm{v}) \mathrm{CvC}$... :

funas «bœuf ", gaji « pièce, chambre », yid « chevreau », mekli « déjeuner », mensi

« dîner ",...

mais aussi brid « chemin », drar = montagne, tri « étoile »,...

10 - La voyelle initiale de la plupart des noms courts est / $\mathrm{u} /$ mais elle est également attestée pour d'autres noms :

m. ufed " genou », ugem « axe », ufes « main », usef « rivière », uzu « mouche »; uglim

"peau»

f. tusa « foie », tugersa « soc », tudeft « laine », tukrimt « cou »

11 - Numération* : Beguinot indique qu'hormis uğun « un», sen « deux» et leur féminin, les autres numéraux sont empruntés à l'arabe. Il semblerait que l'ancienne numération soit uniquement utilisée comme argot. Motylinski (1898) présente par contre un système de numération quinaire basé sur :

uğun, sen, šaret « trois », okkoz « quatre ",

ufes « main = cinq », uyer « lune = trente », temiți « cent »

12 - La forme de base du déictique de proximité suffixé au nom est / $\mathrm{u} /$ comme dans d'autres parlers zénètes : -uh (variante : -uha) sur lequel est formé le pronom déictique : wuh $(a)$ « celui-ci » et $\operatorname{tuh}(a)$ « celle-ci ».

aterras-uh(a) «cet homme » $>$ wuh $(a)$ « celui-ci »

13 - Le nombre : outre les types de pluriels classiques en berbère, les noms propres et certains noms de parenté font leur pluriel à l'aide des particules id ou ind attestées à Ghadamès, en touareg et en chleuh (Chaker 2001). Il existe une forme féminine de cette particule (tind) inconnue ailleurs :

batti « ma tante paternelle », pluriel : tind batti

\section{Système verbal}

14 - Il existe trois thèmes verbaux : le prétérit (accompli), l'aoriste toujours accompagné de la particule $a(d)$ ou $s a(d)$ et l'aoriste intensif (inaccompli). La négation* se fait simplement à l'aide de la particule préverbale wel (et variantes) et postverbale ši (ou š). Il n'y a pas de thème verbal négatif.

15 - Comme pour certains parlers dits zénètes*, on trouve l'apophonie finale -ø / (-i /-u) pour les verbes du type : C (ex : eg « faire »), CC (ex : efk «donner» ou err « rendre») et aC (ex : ar « ouvrir) :

yefku « il a donné », yuru « il a ouvert »

16 - On peut distinguer deux groupes de verbes, l'un avec apparition d'une voyelle $/ \mathrm{u} /$ aux $2^{\mathrm{e}}$ et $3^{\mathrm{e}} \mathrm{p}$. du pluriel de tous les thèmes, y compris l'impératif (thèmes en u) et l'autre sans (thèmes sans $u$ ). Ce phénomène, à distinguer de l'apophonie, est similaire à celui que l'on retrouve à Ghadamès. Exemples :

Encyclopédie berbère, 33 | 2012 
Thèmes en « $u$ »

«jouer », urar : verbe sans apophonie

Impératif $=2 \mathrm{~s}$ urar $/ 2 \mathrm{mpl}$ urarut $/ 2 \mathrm{fpl} \mathrm{urarumet}$

Aoriste $=3 \mathrm{~ms}$ a yurar $/ 1 \mathrm{pl}$ a nurar $/ 2 \mathrm{mpl}$ ad turarum $/ 3 \mathrm{mf}$ ad urarunet

Prétérit $=3 \mathrm{~ms}$ yurar $/ 1 \mathrm{pl}$ nurar $/ 2 \mathrm{mpl}$ turarum $/ 3 \mathrm{mf}$ urarunet

Aoriste intensif $=3 \mathrm{~ms}$ yetturar $/ 1 \mathrm{pl}$ netturar $/ 2 \mathrm{mpl}$ tetturarum $/ 3 \mathrm{mf}$ tturarunet

« donner », efk: verbe avec apophonie $\varnothing /(i / u)$ au prétérit

Impératif $=2 \mathrm{~s}$ efk $/ 2 \mathrm{mpl}$ efkut $/ 2 f p l$ efkumet

Aoriste $=3 \mathrm{~ms}$ a yefk $/ 1 \mathrm{pl} \mathrm{a} \mathrm{nefk} / 2 \mathrm{mpl}$ ad tefkum $/ 3 \mathrm{mf}$ ad efkunet

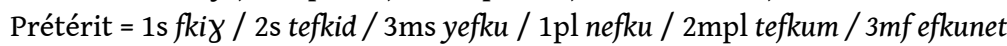

Aoriste intensif $=3 \mathrm{~ms}$ ifekk / $1 \mathrm{pl} \mathrm{nfekk} \mathrm{/} 2 \mathrm{mpl}$ tfekkum / 3mf fekkunet

Thèmes sans « $u$ »

" écrire » ari : verbe avec apophonie a/i au prétérit

Impératif $=2 \mathrm{~s}$ ari $/ 2 \mathrm{mpl}$ arit $/ 2 \mathrm{fpl}$ arimet

Aoriste $=3 \mathrm{~ms}$ a yari $/ 1 \mathrm{pl}$ a nari $/ 2 \mathrm{mpl}$ ad tarim $/ 3 \mathrm{mf}$ ad arinet

Prétérit $=3 \mathrm{~ms}$ yuri $/ 1 \mathrm{pl}$ nuri $/ 2 \mathrm{mpl}$ turim $/ 3 \mathrm{mf}$ urinet

Aoriste intensif $=3 \mathrm{~ms}$ yettari $/ 1 \mathrm{pl}$ nettari $/ 2 \mathrm{mpl}$ tettarim $/ 3 \mathrm{mf}$ ttarinet

17 - Le participe* semble en voie de disparition. On trouve dans Beguinot quelques exemples au prétérit avec la même forme qu'en kabyle, par exemple : $y$ + thème $+n$

aterras lli di-yemlun «l'homme qui m'a dit»

mammu t-yemlun « qui l'a dit?»,

mammu yen zun aterras-uha " qui a tué cet homme?»

à côté de :

aterras lli d-yusû « l'homme qui est venu »

tmetțut lli tekmû « la femme qui est entrée »

Pour les autres thèmes verbaux, c'est toujours la forme conjuguée qui est employée :

aterras lli a di-imel « l'homme qui me dira »

19 - Les verbes de qualité : le nefoussi possède une conjugaison particulière au prétérit pour certains verbes dit d'état ou de qualité. Au singulier, il n'y a qu'une variation de genre et au pluriel, une forme unique.

meššek « être petit »

ms meššek / fs meššekyet; pl. meššuket

Bušil meššek « un garçon petit, un petit garçon » (là où le kabyle emploierait le participe)

La suffixation d'un déictique transforme le syntagme nominal en syntagme prédicatif :

Bušil-uh meššek « ce garçon est petit »

21 L'adjectif issu de ces verbes fournit une forme déterminée ou un syntagme prédicatif nominal :

Bušil ameškan « le petit garçon »

Bušil-uh ameškan « ce garçon le petit » = « c'est le petit garçon »

\section{Prépositions / conjonctions}

22 - La préposition in (variante $n$ ) « vers, à » est employée pour indiquer la destination et l'attribution (là où dans la plupart des autres variétés berbères, c'est la préposition $i$ qui joue ce rôle)

Ugure in Ṭarables « je suis allé à Tripoli »

Yugur in taddart « il alla à la maison »

Yeml'as $n$ bušil « il dit au garçon » (<yemlu-as...) 
Yeml'as in baba-nnes " il dit à son père " (yemlu-as...)

Yefku n uray « il donna au renard » coordination contrairement à la plupart des parlers berbères Nord :

yeszu taddart d iṣar nìt bāb $n$ tmura « il acheta une maison et devint le maître du pays"

ded teml'as... « et elle lui dit... » (<ded temlu-as...)

- La proposition relative : comme à Siwa par exemple, la relative a subi l'influence de l'arabe qui se traduit par l'apparition d'un pronom de rappel :

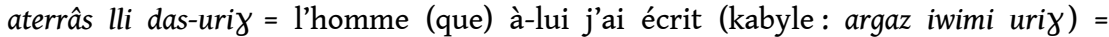
l'homme à qui j'ai écrit

- La particule prédicative $d$ : dans les parlers décrits par Beguinot, elle n'est pas employée dans des prédicats nominaux :

essuq amoqrân di drar $n$ Infusen win Yefren « le plus grand marché dans le Nefoussa est celui de Yefren "

wuha abahlul « c'est un fou » (litt. celui-ci fou)

neččent tbušilin «nous sommes des fillettes » (litt. nous (f.) fillettes)

Cependant, les compléments propositionnels «attributs» de certains verbes: faire, devenir, rendre,... comportent la particule prédicative :

a t-negg d rummî-tnay «nous le considérerons comme (litt. ferons être) notre frère »

\section{Les parlers orientaux (Yefren)}

Aucune étude n'a encore été publiée sur ces parlers, mais sur la base d'une enquête très limitée, il ressort qu'il s'agit bien de parlers assez différents de ceux des variétés occidentales :

- Présence d'une distinction entre un état libre et un état d'annexion;

- Système verbal « classique » (absence des thèmes en $u$ ) et apophonie -ø / $(-i /-a)$;

- Lexique plus proche des parlers zénètes :

« donner » = uš au lieu de efk ; «frère » = uwwa au lieu de rummu ; « un »=iǧen au lieu de uğun, etc.

- Déictique de proximité (suffixé) à forme de base - $a$;

- Particule prédicative $d$ bien attestée dans les énoncés nominaux ;

-Voyelle initiale : même fonctionnement qu'au Mzab ou dans le Rif par exemple :

fus « main ", fud " genou ", suf " rivière ", etc.

- Il semblerait que le participe ait totalement disparu dans ces parlers. 


\section{Note sur la langue du commentaire berbère de la Mudawwana d'Ibn Ghanem}

31 Dans la préface à son étude sur le nefoussi, Beguinot (1942) émet un jugement intéressant sur la langue employée par Chemmakhi (l'informateur de Motylinski (1898)) pour rédiger «le Djebel Nefousa»: il écrit que l'auteur a composé son texte en empruntant des éléments à plusieurs dialectes et a produit ainsi une langue aussi pure que possible, ce qui la rend difficilement compréhensible par la plupart des berbérophones de la région hormis ceux ayant des connaissances d'autres variétés berbères.

Si la langue de Chemmakhi est sans conteste du nefoussi, celle du commentaire - rédigé antérieurement au XV $\mathrm{XV}^{\mathrm{e}}$ siècle - de la mudawwana d'Ibn Ghanem par Abu Zakarya de Yefren (Motylinski 1907) est très différente. Elle n'est certainement pas basée sur la langue décrite par Beguinot (parlers occidentaux du djebel Nefoussa), comme le montre également le " vocabulaire berbère ancien » tiré de ce commentaire et présenté comme un « dialecte du djebel Nefoussa » (Bossoutrot 1900). Un survol du manuscrit permet de faire les observations préliminaires suivantes :

- Existence de spirantes, pas de thèmes verbaux en $u$;

- Emploi du verbe uš « donner » (et non efk);

- nešnin « nous », iğen « un » et non neččen, uğun (Beguinot 1942);

- Pas de noms avec voyelle initiale $u$ (fus et non ufes) ;

- Utilisation de la particule prédicative $d$ en phrase nominale ;

- Présence de thèmes verbaux négatifs en /i/, etc.

En attendant une étude plus complète du manuscrit, on peut seulement formuler l'hypothèse que la langue semble très proche des parlers de Yefren, mais également de ceux de Djerba ou du Mzab.

\section{BIBLIOGRAPHIE}

BEGUINOT Francesco, Il Berbero Nefûsi di Fassâțo. Grammatica, testi raccolti dalla viva voce, vocabolarietti, Roma, Istituto per l'Oriente, 1942

Bossoutrot A., « Vocabulaire berbère ancien (dialecte du djebel Nefoussa) », La revue tunisienne 1900, p. 489-507

BRUGNATELLI V., «D'une langue de contact entre Berbères ibadites », Berber in Contact. Linguistic and Sociolinguistic Perspectives, M. Lafkioui \& V. Brugnatelli (éds), Köln, Rüdiger Köppe Verlag (Berber Studies, vol. 22), p. 39-52

BUSELLI G., « Berber Texts from Jebel Nefûsi (Žemmâri Dialect) », Journal of the Royal African Society, vol. 23, n 92, 1924, p. 285-293

CHAKER S., « Ecriture (graphie arabe) », Encyclopédie Berbère, XVII, 1996, p. 2580-2583 
CHAKER S., «Ida », Encyclopédie Berbère, XXIV, 2001, p. 3619-3620

LOUALI N. \& PHiLIPPSON, G., « Deux systèmes accentuels berbères : le siwi et le touareg 》, Faits de langues, $26: 1,2005$, p. 11-22

LOUALI N., «L'accent en berbère : catégorie grammaticale et démarcation syntaxique ", Nouvelles études berbères. Le verbe et autres articles, K. Naït-Zerrad, R. Vossen \& D. Ibriszimow (éds), Köln, Rüdiger Köppe Verlag (Berber Studies, vol. 8), 2004, p. 67-77

MOTYLINSKI A. de C., « Le manuscrit arabo-berbère de Zouagha découvert par M. Rebillet. Notice sommaire et extraits ", Actes du XIVe Congrès international des Orientalistes, Alger, 1905, Paris, E. Leroux, t. II, 1907, p. 68-78.

MOTYLINSKI A. de C., Le Djebel Nefousa : transcription, traduction française et notes, avec une étude grammaticale, Paris, Ernest Leroux, 1898.

OULD-BRAHAM O., " Sur un nouveau manuscrit ibâdite-berbère. La mudawwana d'Abû Gânim alHurâsânî traduite en berbère au Moyen-Âge ", Etudes et Documents Berbères, 27, 2008, p. 47-71.

PRASSE K.-G., « Le ghadamsi », Encyclopédie Berbère, XX, 1998, p. 3073-3078

INDEX

Mots-clés : Libye, Linguistique, Nefoussa 